\title{
Study of the pion vector form factor and its contribution to the muon $g-2$
}

\author{
Jing-Yu Yi®, Zhong-Yu Wang $\odot$, and C. W. Xiao®* \\ School of Physics and Electronics, Hunan Key Laboratory of Nanophotonics and Devices, \\ Central South University, Changsha 410083, China
}

(Received 2 August 2021; revised 21 November 2021; accepted 28 November 2021; published 21 December 2021)

\begin{abstract}
In the present work, we investigate several theoretical models of the pion vector form factor and aim at getting the best fit for the two-pion cross sections to reduce the uncertainties of the calculation of two-pion contribution to the muon anomalous magnetic moment. Combined with a polynomial description to the pion vector form factor, we obtain the best fit from the Gounaris-Sakurai (or Kühn-Santamaria) model for the experimental data up to $1 \mathrm{GeV}$. As a by-product of the fitting results, the branching ratio of $\omega \rightarrow \pi \pi$ can be extracted as $\operatorname{Br}(\omega \rightarrow \pi \pi)=(1.52 \pm 0.06) \%$, which is consistent with the one of the Particle Data Group. With the best fit of the data, we obtain the muon anomalous magnetic moment from two-pion contribution as $a_{\mu}^{\mathrm{HVP}, \mathrm{LO}}\left(\pi^{+} \pi^{-} \leq 1 \mathrm{GeV}\right)=(497.76 \pm 3.15) \times 10^{-10}$. Our results are consistent with the other works.
\end{abstract}

DOI: 10.1103/PhysRevD.104.116017

\section{INTRODUCTION}

The muon magnetic moment is an important and long historical issue in particle physics [1-7]. Using Dirac theory, the gyromagnetic ratio $g_{\mu}$ is predicted as $g_{\mu}=2$ for the structureless and spin- $\frac{1}{2}$ muon. In fact, due to the developments of the experiments and theories, it is found that $g_{\mu}$ is slightly greater than 2 , which can be referred to as the anomalous magnetic moment $a_{\mu}=\left(g_{\mu}-2\right) / 2$. As already known, it is Schwinger's value of $a_{\mu}=\alpha /(2 \pi) \simeq$ 0.00116 from one-loop QED radiative corrections, which is universal for all leptons. More discussions for that can be found in the reviews of Refs. [8,9]. The anomalous magnetic moment of the muon $a_{\mu}$ is experimentally and theoretically known to very high accuracy. Its measurement at Brookhaven National Laboratory was reported as $[10,11]$

$$
a_{\mu}^{\exp }=(11659209.1 \pm 5.4 \pm 3.3) \times 10^{-10},
$$

where the errors were given by statistical and systematic uncertainties, respectively. The standard model (SM) prediction was given by [12]

$$
a_{\mu}^{\mathrm{SM}}=(11659183.1 \pm 4.0 \pm 2.6 \pm 0.1) \times 10^{-10} .
$$

And thus, the difference between the experiment and theory is

\footnotetext{
*xiaochw@csu.edu.cn
}

Published by the American Physical Society under the terms of the Creative Commons Attribution 4.0 International license. Further distribution of this work must maintain attribution to the author(s) and the published article's title, journal citation, and DOI. Funded by SCOAP ${ }^{3}$.

$$
\Delta a_{\mu}=a_{\mu}^{\mathrm{exp}}-a_{\mu}^{\mathrm{SM}}=(26.0 \pm 7.9) \times 10^{-10},
$$

where one can see that there is a discrepancy of about $3.3 \sigma$ between the measured value and the full standard model prediction. But this discrepancy has been updated with the recent results in both theory calculations and experimental measurements. The latest measurement of the anomalous magnetic moment of the muon was performed at Fermilab National Accelerator Laboratory Muon $g-2$ Experiment [13], given by

$$
a_{\mu}^{\exp }=(11659204.0 \pm 5.4) \times 10^{-10} .
$$

Combined with the measurement at Brookhaven above, one can easily get the experimental average of

$$
a_{\mu}^{\exp }=(11659206.1 \pm 4.1) \times 10^{-10} .
$$

Note that the reported results of Ref. [10] were used for this average. The latest SM prediction was given by the recent review of the white paper (WP) [14]

$$
a_{\mu}^{\mathrm{SM}}=(11659181.0 \pm 4.3) \times 10^{-10} .
$$

Therefore, the difference between the experiment and the theory is updated as

$$
\Delta a_{\mu}^{\text {new }}=a_{\mu}^{\text {exp }}-a_{\mu}^{\mathrm{SM}}=(25.1 \pm 5.9) \times 10^{-10},
$$

which leads to a discrepancy of $4.2 \sigma$. This discrepancy possibly hints the new physics beyond SM and draws much theoretical attention [15-31]. More discussions can be found in Refs. [28,32] for new physics beyond SM and 
the latest review in Ref. [33] for recent status, and references therein.

So, it is crucial to know the prediction of the SM as precisely as possible. The prediction of the $\mathrm{SM} a_{\mu}^{\mathrm{SM}}$ can be divided into several different contributions [14,34],

$$
a_{\mu}^{\mathrm{SM}}=a_{\mu}^{\mathrm{QED}}+a_{\mu}^{\mathrm{had}}+a_{\mu}^{\mathrm{weak}},
$$

where $a_{\mu}^{\mathrm{QED}}$ is the pure electromagnetic contribution, $a_{\mu}^{\mathrm{had}}$ is the hadronic contribution, and $a_{\mu}^{\text {weak }}$ accounts for the electroweak corrections due to the exchange of the weak interacting bosons. At present, $a_{\mu}^{\mathrm{QED}}$ was calculated with high accuracy up to five-loop order [35-38], and $a_{\mu}^{\text {weak }}$ was also done up to two-loop order [39-41], which were given by

$$
\begin{gathered}
a_{\mu}^{\mathrm{QED}}=(11658471.8931 \pm 0.0104) \times 10^{-10}, \\
a_{\mu}^{\text {weak }}=(15.36 \pm 0.10) \times 10^{-10},
\end{gathered}
$$

where one can see the reviews in Refs. $[14,33,34]$ for more details. Thus, the large uncertainties of $a_{\mu}^{\mathrm{SM}}$ mainly come from the hadronic part of $a_{\mu}^{\text {had }}$ due to the confinement and nonperturbative properties in the low-energy region, which can be divided into two parts, one part from hadronic lightby-light (HLbL) scattering $\left(a_{\mu}^{\mathrm{HLbL}}\right)$ and the other one from the hadronic vacuum polarization (HVP) contribution $\left(a_{\mu}^{\mathrm{HVP}}\right)$.

For the HLbL contribution [42-47], the phenomenological estimation was given by the WP [14],

$$
a_{\mu}^{\mathrm{HLbL}}=(9.2 \pm 1.9) \times 10^{-10},
$$

which was consistent with lattice QCD calculations $a_{\mu}^{\mathrm{HLbL}}=$ $(7.87 \pm 3.06 \pm 1.77) \times 10^{-10}$ [48] and $a_{\mu}^{\mathrm{HLbL}}=(10.68 \pm$ $1.47) \times 10^{-10}$ [49] within the uncertainties. Recently (after the WP), with a model-independent method, the effects of short-distance constraints on the $a_{\mu}^{\mathrm{HLbL}}$ were evaluated in Ref. [50] by considering the known states below $1 \mathrm{GeV}$, which obtained $a_{\mu}^{\mathrm{HLLL}}=(0.91 \pm 0.50) \times 10^{-10}$ for the contribution of pseudoscalar ground states and $a_{\mu}^{\mathrm{HLbL}}=$ $(0.26 \pm 0.15) \times 10^{-10}$ for the contribution of isovector parts. The short-distance expansion for the four-point function was derived in Ref. [51] via a systematic operator product expansion, where it was found that the contribution of the massless quark loop in leading order to the $a_{\mu}^{\mathrm{HLbL}}$ was dominant and the contributions from higher order were estimated to be small. In the further work of Ref. [52], the perturbative QCD correction to the massless quark loop was computed, and the authors found that the correction up to two loops was a quite small contribution to the $a_{\mu}^{\mathrm{HLbL}}$. Reference [53] discussed the short-distance constraints to the calculation of $a_{\mu}^{\mathrm{HLbL}}$ from the contribution of the axial-vector mesons. Employing resonance chiral theory, the axial-vector contribution to the $a_{\mu}^{\mathrm{HLbL}}$ was discussed in Ref. [54] with a small result of $a_{\mu}^{\mathrm{HLbL} ; \mathrm{A}}=\left(0.8_{-0.8}^{+3.5}\right) \times 10^{-11}$. On the other hand, with a warped five-dimensional model, Ref. [55] considered the contributions of pseudoscalar and axial-vector resonances to the $a_{\mu}^{\mathrm{HLbL}}$ and obtained a value of $a_{\mu}^{\mathrm{HLbL} ; \mathrm{P}+\mathrm{A}}=(12.5 \pm 1.5) \times 10^{-10}$ with a much larger role for the axial-vector contribution. In Ref. [56], the transition form factors of the resonance $f_{1}(1285)$ were analyzed in detail with the framework of vector meson dominance due to its contribution to the HLbL scattering; see more details in Ref. [57]. Using dispersion relations, Ref. [58] considered the contribution of scalar resonances to the $a_{\mu}^{\mathrm{HLbL}}$ and obtained an estimate of $a_{\mu}^{\mathrm{HLbL}}[\mathrm{scalar}]=$ $(-0.9 \pm 0.1) \times 10^{-10}$. Moreover, several models for the short-distance constraints to the calculation of $a_{\mu}^{\mathrm{HLbL}}$ were investigated in detail in Ref. [59], in which the perturbative QCD correction was also taken into account and the result of the perturbative corrections to the operator product expansion was updated as $a_{\mu}^{\mathrm{HLbL}}=(1.3 \pm 0.5) \times 10^{-10}$.

On the other hand, at the current status, the total HVP contribution was estimated as [14,33]

$$
a_{\mu}^{\mathrm{HVP}}=(684.5 \pm 4.0) \times 10^{-10},
$$

which included the leading-order (LO) [12,60], next-toleading-order [60], and next-next-to-leading-order [61] contributions. In fact, the dominant one is the LO part, given by the data-driven calculations [12,14,60,62-65]

$$
a_{\mu}^{\mathrm{HVP}, \mathrm{LO}} \underset{\mathrm{DaDr}}{ }=(693.1 \pm 4.0) \times 10^{-10},
$$

which is overlapped with the lattice world average for the total LO HVP contribution [14],

$$
a_{\mu}^{\mathrm{HVP}, \mathrm{LO}}=(711.6 \pm 18.4) \times 10^{-10} .
$$

But the recent calculation of lattice QCD for the LO HVP contribution was reported as [66]

$$
a_{\mu}^{\mathrm{HVP}, \mathrm{LO}}=(707.5 \pm 5.5) \times 10^{-10},
$$

with high accuracy, which is a bit smaller than the one obtained in Ref. [67], $(714 \pm 27 \pm 13) \times 10^{-10}$. These new lattice results lead the SM prediction to be in agreement with the current experimental measurement and the new physics to be questionable.

One thing should be mentioned: the LO part from the data-driven calculations in Eq. (13) is only taking the $e^{+} e^{-}$annihilation data into account, since the result from $\tau$ decay data is not precise enough at present. With the results of Ref. [68], there was still $2.2 \sigma$ discrepancy between the $e^{+} e^{-}$-based and $\tau$-based results. Recently, with resonance chiral theory supplemented by dispersion 
relations, Ref. [69] studied the pion vector form factor (PVFF) using the experimental data of $\tau$ decay from Belle and recent $B A B A R$ measurements. Based on the results of Ref. [69], the $a_{\mu}^{\mathrm{HVP}, \mathrm{LO}}$ was extracted from the $\tau$ decay data of $\tau^{-} \rightarrow \pi^{-} \pi^{0} \nu_{\tau}$ in a further work [70], obtained $a_{\mu}^{\mathrm{HVP}, \mathrm{LO}}=$ $\left(705.7_{-4.1}^{+4.0}\right) \times 10^{-10}$ and $a_{\mu}^{\mathrm{HVP}, \mathrm{LO}}=\left(700.7_{-5.2}^{+6.1}\right) \times 10^{-10}$ for different strategies. Using a framework of the hidden local symmetry model combined with appropriate symmetry breaking mechanisms, both the $e^{+} e^{-}$annihilation and $\tau$ decay data were analyzed in Ref. [71], in which a value of $a_{\mu}^{\mathrm{HVP}, \mathrm{LO}}=(686.65 \pm 3.01) \times 10^{-10}$ with the uncertainties of $\rho-\gamma$ mixing was reported and a further result was updated in the recent work of Ref. [72].

As one can see, for the SM prediction $a_{\mu}^{\mathrm{SM}}$, the hadronic part $a_{\mu}^{\text {had }}$ still has large uncertainties, especially for the one $a_{\mu}^{\mathrm{HVP}}$. Recently, to solve the inverse problem to the dispersion relation, a value for the HVP contribution was obtained as $a_{\mu}^{\mathrm{HVP}}=\left(641_{-63}^{+65}\right) \times 10^{-10}$ in Ref. [73]. Based on the chiral perturbation theory, Ref. [74] discussed that the finite-volume corrections to $a_{\mu}^{\mathrm{HVP}}$ could be precisely evaluated, where once all low-energy constants were already known. Reference [75] investigated the potential impact on the electroweak fits of the tensions between the current determinations of the HVP contributions to the $a_{\mu}$, based on either phenomenological calculations or lattice QCD calculations. Note that, taking into account the measurement of the Higgs mass, the impact of HVP on $a_{\mu}^{\mathrm{SM}}$ and the global fits to electroweak precision data was studied in Ref. [76], in which some options for physics beyond SM were discussed.

To reduce the uncertainties of the part $a_{\mu}^{\mathrm{HVP}}$, indeed, the accurate evaluations must rely on the corresponding cross section measurements for the normal data-driven calculations. Combined with the effective Lagrangian, an iterated global fit scheme was adopted in Ref. [77] to reduce the uncertainties for the description of the $e^{+} e^{-} \rightarrow \pi^{+} \pi^{-}$ annihilation data up to $1.05 \mathrm{GeV}$. With a dispersive representation of the PVFF, different constraints on the two-pion contribution were examined for its effects on the $a_{\mu}^{\mathrm{HVP}}$ in Ref. [78], in which a value of $a_{\mu}^{\mathrm{HVP}}(\pi \pi \leq 1 \mathrm{GeV})=$ $497.0(1.4) \times 10^{-10}$ was gotten in one case of their fits. Using a parametrization-free formalism based on analyticity and unitarity, the PVFF and its contribution to the $a_{\mu}^{\mathrm{HVP}}$ were investigated in Refs. [79-81] for the energy range around the $\rho(770)$ resonance. As already known, about $73 \%$ of the LO hadronic contribution and about $60 \%$ of the total uncertainty are given by the cross section of $e^{+} e^{-}$ annihilated to the $\pi^{+} \pi^{-}(\gamma)$ final states, which are dominated by the $\rho(770)$ resonance. Therefore, it is important to study the $e^{+} e^{-} \rightarrow \pi^{+} \pi^{-}$annihilation, which always relates to the PVFF. Thus, in the present work, we study several theoretical models of the PVFF and aim at finding out the best fit of the $\pi^{+} \pi^{-}$scattering cross sections. In the next section, we first introduce the calculation of $a_{\mu}^{\mathrm{HVP}, \mathrm{LO}}$ with the data-driven approach briefly. In the following, we discuss several phenomenological models of the PVFF, combined with a polynomial description, or equivalently how to take into account the contribution of the $\rho(770)$ resonance. Then, we obtain the results from fitting the PVFF data of the collaborations Orsay, DM1, OLYA, CMD1, CMD2, BABAR, BESIII, KLOE, SND, and so on. And thus, we perform a calculation of $a_{\mu}^{\mathrm{HVP}, \mathrm{LO}}$ up to $1 \mathrm{GeV}$. At the end is our conclusion.

\section{MUON $(g-2)$ CALCULATION WITH DISPERSION RELATION}

As we discussed above, the theoretical prediction of $a_{\mu}^{\mathrm{SM}}$ has large uncertainties from the parts of hadronic contribution $a_{\mu}^{\text {had }}$. Because of the confinement, the nonperturbative properties become dominant in the low-energy region, where the quarks are confined inside hadrons. Therefore, perturbative QCD fails to evaluate the hadronic (quark and gluon) loop contributions to the $a_{\mu}^{\mathrm{SM}}$ precisely. In principle, one can do the calculation of $a_{\mu}^{\text {had }}$ from first-principle calculation in lattice QCD. But most of the evaluations in lattice QCD are still not precise enough, except for the recent one of Ref. [66]. Alternatively, the HVP contributions $a_{\mu}^{\mathrm{HVP}}$ can be calculated with the data-driven approach, which uses the dispersion relation together with the optical theorem and experimental data. Thus, the LO HVP contribution to the $a_{\mu}^{\mathrm{SM}}$ can be calculated via a dispersion relation using the measured cross sections of $e^{+} e^{-} \rightarrow$ hadrons [82]

$$
a_{\mu}^{\mathrm{HVP}, \mathrm{LO}}=\frac{1}{\pi} \int_{0}^{\infty} \frac{\mathrm{d} s}{s} \operatorname{Im} \Pi^{(\mathrm{H})}(s) K(s),
$$

where the kernel function is given by

$$
\begin{aligned}
K(s)= & \left(\frac{\alpha}{\pi}\right)\left\{\frac{1}{2} x^{2}\left(2-x^{2}\right)+(1+x)^{2}\left(1+x^{2}\right)\right. \\
& \left.\times \frac{\ln (1+x)-x+\frac{1}{2} x^{2}}{x^{2}}+\frac{1+x}{1-x} x^{2} \ln x\right\},
\end{aligned}
$$

with the definitions

$\beta_{\mu}=\sqrt{1-\left(4 m_{\mu}^{2} / s\right)}, \quad x=\left(1-\beta_{\mu}\right) /\left(1+\beta_{\mu}\right)$

and the electromagnetic coupling taken as $\alpha=e^{2} /(4 \pi) \approx$ 1/137.036 from the Particle Data Group (PDG) [34]. Note that $s$ is the total energy of two-body system, $s \equiv\left(p_{1}+p_{2}\right)^{2}$. With the optical theorem, the imaginary part of the vacuum polarization amplitude $\operatorname{Im} \Pi^{(\mathrm{H})}(s)$ can be expressed in terms of the total cross section of the electronpositron annihilation into hadrons, 


$$
\sigma_{\text {tot }}\left(\mathrm{e}^{+} \mathrm{e}^{-} \rightarrow \text { hadrons }\right)=\frac{4 \pi^{2} \alpha}{s} \frac{1}{\pi} \operatorname{Im} \Pi^{(\mathrm{H})}(s) .
$$

Thus, one can deduce

$$
a_{\mu}^{\mathrm{HVP}, \mathrm{LO}}=\frac{1}{4 \pi^{2} \alpha} \int_{m_{\pi}^{2}}^{\infty} \mathrm{d} s \sigma_{\mathrm{tot}}\left(\mathrm{e}^{+} \mathrm{e}^{-} \rightarrow \text { hadrons }\right) K(s),
$$

which uses the measured bare cross sections for the annihilation $\mathrm{e}^{+} \mathrm{e}^{-} \rightarrow$ hadrons as inputs and where the lower limit of the dispersion integral is in fact the $\pi^{0} \gamma$ cut. One should keep in mind that the experimentally measured cross sections are the dressed cross sections, where the bare cross sections can be corrected by the running of the coupling constant $\alpha(s)$. In fact, this correction has always been done in the experimental data reported. Thus, the corresponding cross section measurements play a key role in the accurate evaluation of $a_{\mu}^{\text {had,LO }}$.

Note that the kernel function $K(s)$ decreases monotonically with increasing $s$, so it gives strong weight to the lowenergy part of the integral, where about $73 \%$ of the LO hadronic contributions are given by the cross section of $\pi^{+} \pi^{-}(\gamma)$ final states, dominated by the $\rho(770)$ resonance. In the present work, we focus on the energy region of about $1 \mathrm{GeV}$, which is mainly contributed by the $\pi^{+} \pi^{-}(\gamma)$ final states. The total cross section contributed by two-pion final states is given by ${ }^{1}$

$$
\sigma_{\text {tot }}\left(\mathrm{e}^{+} \mathrm{e}^{-} \rightarrow \pi^{+} \pi^{-}\right)=\frac{1}{3} \pi \alpha^{2} \frac{1}{s} \beta_{\pi}^{3}(s)\left|F_{\pi}(s)\right|^{2},
$$

where $F_{\pi}(s)$ is the PVFF and the pion phase is defined as $\beta_{\pi}(s)=\sqrt{1-4 m_{\pi}^{2} / s}$. Then, it is important to study the model of PVFF; see the discussion in the next section. Thus, the two-pion contribution to the anomalous magnetic moment of the muon can be written as

$a_{\mu}^{\mathrm{HVP}, \mathrm{LO}}\left(\pi^{+} \pi^{-}\right)=\frac{\alpha}{12 \pi} \int_{4 m_{\pi}^{2}}^{\infty} \frac{\mathrm{d} s}{s} \beta_{\pi}^{3}(s)\left|F_{\pi}(s)\right|^{2} K(s)$.

\section{MODEL FOR THE PION FORM FACTOR}

In the present work, we are interested in the experimental data at a center-of-mass energy below $1 \mathrm{GeV}$, which is around the energy region corresponding to the $\rho$ resonance. For the cross section of $\mathrm{e}^{+} \mathrm{e}^{-} \rightarrow \pi^{+} \pi^{-}$, it can be associated with the PVFF, see Eq. (21), which can be defined as

$$
\left\langle\pi^{+}\left(p^{\prime}\right) \pi^{-}(p)\left|J_{\mu}(0)\right| 0\right\rangle=\left(p^{\prime}-p\right)_{\mu} F_{\pi}(s),
$$

\footnotetext{
${ }^{1}$ In fact, the two-pion cross section is inclusive of final-state radiation effects and exclusive of all vacuum polarization effects in the experimental measurements.
}

with $s=\left(p^{\prime}+p\right)^{2}$ and $J_{\mu}$ the vector-isovector current. For our case of energy range below $1 \mathrm{GeV}$, in terms of the pion $P$-wave phase shift $\delta_{11}(s)$, the PVFF fulfills the following discontinuity condition:

$$
\operatorname{Im} F_{\pi}(s)=F_{\pi}(s) \sin \delta_{11}(s) e^{-i \delta_{11}(s)} \Theta\left(s-4 m_{\pi}^{2}\right) .
$$

With a once-subtracted dispersion relation, the solution of Eq. (24) can be written into a general ansatz $[83,84]$,

$$
F_{\pi}(s)=P(s) \Omega(s)
$$

where $P(s)$ is a polynomial and $\Omega(s)$ is the Omnés function [85]. Note that the solution for higher subtracted dispersion relation can be referred to Refs. [86-88] for more discussions and applications. Especially, using thricesubtracted dispersion relation, Ref. [86] made a good description of the PVFF experimental data up to $\sqrt{s} \simeq$ 1.2 GeV with two subtraction constants and evaluated the two-pion contribution to the $a_{\mu}^{\mathrm{HVP}, \mathrm{LO}}$. Furthermore, this ansatz was extrapolated to the radiative decays of $\eta^{(\prime)} \rightarrow$ $\pi^{+} \pi^{-} \gamma[89,90]$, where a linear polynomial was used,

$$
P(s)=1+\alpha s,
$$

with $\alpha$ a free parameter, determined from the data. Indeed, the linear behavior was clearly shown in the results of Refs. [89,90] for the data of the PVFF below $1 \mathrm{GeV}$. A new parametrization to the PVFF for a full energy range can be found in Ref. [91], in which the isospin violation mechanism was also considered, such as the mixing effects of $\rho-\omega$ and $\omega-\phi$. Besides, the Omnés function is given by

$$
\Omega(s)=\exp \left(\frac{s}{\pi} \int_{4 m_{\pi}^{2}}^{\infty} \frac{d s^{\prime}}{s^{\prime}} \frac{\delta_{11}\left(s^{\prime}\right)}{s^{\prime}-s-i \epsilon}\right)
$$

where the phase shift $\delta_{11}(s)$ can be taken from the Madrid model's results [92]. In Ref. [92], the phase shift for $s^{1 / 2} \leq$ $2 m_{K}$ fulfilled

$$
\begin{aligned}
\cot \delta_{11}(s) & =\frac{s^{1 / 2}}{2 k^{3}}\left(m_{\rho}^{2}-s\right)\left\{\frac{2 m_{\pi}^{3}}{m_{\rho}^{2} \sqrt{s}}+B_{0}+B_{1} w(s)\right\}, \\
w(s) & =\frac{\sqrt{s}-\sqrt{s_{0}-s}}{\sqrt{s}+\sqrt{s_{0}-s}}, \quad s_{0}^{1 / 2}=1.05 \mathrm{GeV},
\end{aligned}
$$

where the $\rho$ mass was fixed to $m_{\rho}=773.6 \mathrm{MeV}$, the other masses were taken as $m_{\pi}=139.57 \mathrm{MeV}$, $m_{K}=496 \mathrm{MeV}, m_{\eta}=547.51 \mathrm{MeV}$, and the central-mass momentum in the two-pion final states $k=\sqrt{s-4 m_{\pi}^{2}} / 2$. For $2 m_{K} \leq s^{1 / 2} \leq 1420 \mathrm{MeV}$, one can have 
$\delta_{11}(s)=\lambda_{0}+\lambda_{1}\left(\sqrt{s} / 2 m_{K}-1\right)+\lambda_{2}\left(\sqrt{s} / 2 m_{K}-1\right)^{2}$,

where $\lambda_{0}$ is fixed from the value of $\delta_{11}\left(4 m_{K}^{2}\right)$ obtained from the low-energy parametrization so that the phase shift is continuous. Besides, the parameters of $B_{0}, B_{1}, \lambda_{1}$, and $\lambda_{2}$ were taken from the two sets of fitting results in Ref. [92]. For higher energy, we choose the phase shifts close to $\pi$ smoothly, written as

$$
\delta_{11}(s)=\pi-\frac{a}{b-s},
$$

where the coefficients $a$ and $b$ are determined with the value of $\delta_{11}\left(s_{0}\right)\left(\sqrt{s_{0}}=1420 \mathrm{MeV}\right.$ for example, in fact, we take $\sqrt{s_{0}}=1300 \mathrm{MeV}$ for the best results) to make it continuous and also keep the derivative at $s=s_{0}$.

In fact, as discussed above, the data for the cross section of two pions below $1 \mathrm{GeV}$ are dominated by the contribution of the $\rho$ resonance. ${ }^{2}$ Indeed, the Omnés function $\Omega(s)$ in Eq. (25) is mainly contributed by the pion $p$-wave phase shift; see Eq. (27). In the present work, we investigate how to include the contribution of $\rho$ to get better description of the experimental data. Thus, we want to know the effects of different models for the part of Omnés function. Note that the energy region of $1 \mathrm{GeV}$ is safely below the inelastic threshold; see the discussions in Ref. [95]. First, we use the model of Heyn and Lang (HL) [96],

$\Omega(s)=\frac{c+m_{\pi}^{2} g(0)}{\tilde{s}_{p}} \frac{\tilde{s}_{p}-s}{a s^{2}+b s+c-\left(s-4 m_{\pi}^{2}\right) g(s) / 4}$,

where the function $g(s)$ is given by the one-pion-loop diagram in the self-energy of $\rho$ resonance,

$$
\begin{aligned}
g(s) & =-\frac{1}{\pi} u \ln \frac{1+u}{1-u}+i u, \quad u=\sqrt{1-4 m_{\pi}^{2} / s}, \quad s \geq 4 m_{\pi}^{2} \\
& =-\frac{2}{\pi} u \arctan \frac{1}{u}, \quad u=\sqrt{4 m_{\pi}^{2} / s-1}, \quad 0 \leq s \leq 4 m_{\pi}^{2} \\
& =-\frac{1}{\pi} u \ln \frac{u+1}{u-1}, \quad u=\sqrt{1-4 m_{\pi}^{2} / s}, \quad s<0, \\
g(0) & =-2 / \pi,
\end{aligned}
$$

and the parameters $a, b$, and $c$ are free. Besides, $\tilde{s}_{p}$ is the value of the zero of the denominator and can be determined from $a, b$, and $c$, using the condition $f(s)=0$, reading

$$
f(s)=a s^{2}+b s+c-\left(s-4 m_{\pi}^{2}\right) g(s) / 4=0 .
$$

Second, as discussed above, the Omnés function is mainly considered the resonance contribution of $\rho$. Thus, using the vector meson dominance approach, one can replace the Omnés function with the simple BreitWigner (BW) form (BW1),

$$
\Omega(s) \rightarrow B W 1=-\frac{M_{\rho}^{2}}{s-M_{\rho}^{2}+i M_{\rho} \Gamma_{\rho}},
$$

with $M_{\rho}$ and $\Gamma_{\rho}$ as free parameters for the $\rho$ meson, which are also fitted by the experimental data. Furthermore, due to the large $\rho$ width, one can use the more common one (BW2) [95],

$$
\Omega(s) \rightarrow B W 2=-\frac{M_{\rho}^{2}}{s-M_{\rho}^{2}-i \sqrt{s} \Gamma(s)},
$$

where the energy-dependent decay width is given by

$$
\Gamma(s)=\Gamma_{\rho}\left[\frac{p(s)}{p\left(M_{\rho}^{2}\right)}\right]^{3} \frac{M_{\rho}^{2}}{s}
$$

with the pion momentum

$$
p(s)=\frac{1}{2} \sqrt{s} \beta_{\pi}(s)=\frac{\sqrt{s-4 m_{\pi}^{2}}}{2} .
$$

Third, for taking into account more precisely the finitewidth corrections, one can also use the method of Gounaris and Sakurai (GS) [97], written as

$$
\Omega(s) \rightarrow B W^{\mathrm{GS}}(s)=\frac{-\left(M_{\rho}^{2}+d M_{\rho} \Gamma_{\rho}\right)}{s-M_{\rho}^{2}-\Gamma_{\rho}\left(M_{\rho}^{2} / p_{\rho}^{3}\right)\left[p^{2}\left(h-h_{\rho}\right)-\left(s-M_{\rho}^{2}\right) p_{\rho}^{2} h_{\rho}^{\prime}\right]+i M_{\rho} \Gamma_{\rho}(s)},
$$

where

\footnotetext{
${ }^{2}$ Note that a new expression for the PVFF was proposed in Ref. [93], in which the contributions from the loops of $\pi^{+} \pi^{-}$and $K \bar{K}$ and higher $\rho$ resonances were considered and which described the data well in the range from -10 to $1 \mathrm{GeV}$ and was extrapolated to the energy up to $3 \mathrm{GeV}$ in the further work of Ref. [94].
} 


$$
\begin{gathered}
p=\left(s / 4-m_{\pi}^{2}\right)^{1 / 2}, \quad h(s)=\frac{2 p}{\pi \sqrt{s}} \ln \left(\frac{\sqrt{s}+2 p}{2 m_{\pi}}\right), \quad s \geq 4 m_{\pi}^{2}, \\
p=i\left(m_{\pi}^{2}-s / 4\right)^{1 / 2}, \quad h(s)=\frac{2 p i}{\pi \sqrt{s}} \operatorname{arccot}\left(\frac{s}{4 m_{\pi}^{2}-s}\right)^{1 / 2}, \quad 0 \leq s<4 m_{\pi}^{2}, \\
p_{\rho}=p\left(M_{\rho}^{2}\right), \quad h_{\rho}=h\left(M_{\rho}^{2}\right), \quad \Gamma_{\rho}(s)=\Gamma_{\rho}\left(\frac{p}{p_{\rho}}\right)^{3} \frac{M_{\rho}}{\sqrt{s}}, \quad h_{\rho}^{\prime}=\left.h^{\prime}(s)\right|_{s=M_{\rho}^{2}},
\end{gathered}
$$

and $d$ is fixed in terms of the masses $M_{\rho}$ and $m_{\pi}$,

$$
d=\frac{3}{\pi} \frac{m_{\pi}^{2}}{p_{\rho}^{2}} \ln \left(\frac{M_{\rho}+2 p_{\rho}}{2 m_{\pi}}\right)+\frac{M_{\rho}}{2 \pi p_{\rho}}-\frac{m_{\pi}^{2} M_{\rho}}{\pi p_{\rho}^{3}} .
$$

Note that, in this GS model, one can also determine the $M_{\rho}$ and $\Gamma_{\rho}$ from the fits. Thus, in BABAR's paper [98], its form was changed equivalently as

$$
B W^{\mathrm{GS}}\left(s, M_{\rho}, \Gamma_{\rho}\right)=\frac{M_{\rho}^{2}\left[1+d\left(M_{\rho}\right) \Gamma_{\rho} / M_{\rho}\right]}{M_{\rho}^{2}-s+f\left(s, M_{\rho}, \Gamma_{\rho}\right)-i M_{\rho} \Gamma\left(s, M_{\rho}, \Gamma_{\rho}\right)},
$$

where

$$
\begin{aligned}
\Gamma\left(s, M_{\rho}, \Gamma_{\rho}\right)= & \Gamma_{\rho} \frac{s}{M_{\rho}^{2}}\left[\frac{\beta_{\pi}(s)}{\beta_{\pi}\left(M_{\rho}^{2}\right)}\right]^{3}, \\
d\left(M_{\rho}\right)= & \frac{3}{\pi} \frac{m_{\pi}^{2}}{k^{2}\left(M_{\rho}^{2}\right)} \ln \left[\frac{M_{\rho}+2 k\left(M_{\rho}^{2}\right)}{2 m_{\pi}}\right] \\
& +\frac{M_{\rho}}{2 \pi k\left(M_{\rho}^{2}\right)}-\frac{m_{\pi}^{2} M_{\rho}}{\pi k^{3}\left(M_{\rho}^{2}\right)}, \\
f\left(s, M_{\rho}, \Gamma_{\rho}\right)= & \frac{\Gamma_{\rho} M_{\rho}^{2}}{k^{3}\left(M_{\rho}^{2}\right)}\left[k^{2}(s)\left(h(s)-h\left(M_{\rho}^{2}\right)\right)\right. \\
& \left.+\left(M_{\rho}^{2}-s\right) k^{2}\left(M_{\rho}^{2}\right) h^{\prime}\left(M_{\rho}^{2}\right)\right],
\end{aligned}
$$

with the pion phase $\beta_{\pi}(s)$ defined as in the last section, and

$$
\begin{aligned}
& k(s)=\frac{1}{2} \sqrt{s} \beta_{\pi}(s), \\
& h(s)=\frac{2}{\pi} \frac{k(s)}{\sqrt{s}} \ln \left(\frac{\sqrt{s}+2 k(s)}{2 m_{\pi}}\right),
\end{aligned}
$$

and $h^{\prime}(s)$ is the derivative of $h(s)$.

Moreover, similar to the GS model, there is another form presented by Kühn and Santamaria (KS) [99],

$$
B W^{K S}(s)=\frac{M_{\rho}^{2}+\Gamma_{\rho} M_{\rho} d^{\prime}}{M_{\rho}^{2}-s+H(s)-i \sqrt{s} \Gamma_{\rho}(s)},
$$

where

$$
\begin{gathered}
H(s)=\hat{H}(s)-\hat{H}\left(M_{\rho}^{2}\right)-\left(s-M_{\rho}^{2}\right) \hat{H}^{\prime}\left(M_{\rho}^{2}\right), \\
\hat{H}(s)=\frac{\Gamma_{\rho} M_{\rho}^{2}}{p_{\rho}^{3}}\left(s / 4-m_{\pi}^{2}\right) h(s), \\
h(s)= \begin{cases}\frac{1}{2 \pi}\left(1-\frac{4 m_{\pi}^{2}}{s}\right)^{1 / 2} \ln \left(\frac{1+\left(1-\frac{4 m_{\pi}^{2}}{s}\right)^{1 / 2}}{1-\left(1-\frac{m_{\pi}^{2}}{s}\right)^{1 / 2}}\right), & 4 m_{\pi}^{2} \leq s \\
\frac{i}{2 \pi}\left(\frac{4 m_{\pi}^{2}}{s}-1\right)^{1 / 2} \ln \left(\frac{i\left(\frac{4 m_{\pi}^{2}}{s}-1\right)^{1 / 2}+1}{i\left(\frac{4 m_{\pi}^{2}}{s}-1\right)^{1 / 2}-1}\right), & 0 \leq s \leq 4 m_{\pi}^{2}\end{cases}
\end{gathered}
$$

$$
\begin{gathered}
d^{\prime}=\frac{3}{2 \pi} \frac{m_{\pi}^{2}}{p_{\rho}^{2}} \ln \left(\frac{M_{\rho}+2 p_{\rho}}{M_{\rho}-2 p_{\rho}}\right)+\frac{M_{\rho}}{2 \pi p_{\rho}}-\frac{m_{\pi}^{2} M_{\rho}}{\pi p_{\rho}^{3}}, \\
p(s)=\frac{1}{2}\left(s-4 m_{\pi}^{2}\right)^{1 / 2}, \quad p_{\rho}=\left(M_{\rho}^{2}-4 m_{\pi}^{2}\right)^{1 / 2} / 2 .
\end{gathered}
$$

Finally, since $e^{+} e^{-}$annihilation data have the $\rho-\omega$ mixing effects, we should take into account this effect as is done in Refs. [12,100],

$$
P(s)=1+\alpha s+\frac{\kappa s}{m_{\omega}^{2}-s-i m_{\omega} \Gamma_{\omega}},
$$

where we take $m_{\omega}=782.65 \mathrm{MeV}$ and $\Gamma_{\omega}=8.49 \mathrm{MeV}$ from the PDG [34] and $\kappa$ is a free parameter containing the information of $\omega \pi \pi$ coupling; see our results later, where more discussions can be referred to Ref. [100]. Note that Eq. (54) fulfills $P(0)=1$, which guarantees the condition $F_{\pi}(0)=1$, except for the BW1 model. Indeed, the BW1 model is the typical one of the vector meson dominance, which is known to violate the condition [101]. Furthermore, in general, one can also float the parameters $m_{\omega}$ and $\Gamma_{\omega}$ in Eq. (54) as is done in Refs. [12,100]. But as found in Ref. [12], a value of $m_{\omega}=(782.0 \pm 0.1) \mathrm{MeV}$ was obtained in the fitting results with all the experimental data, which is not much different from the PDG value we used. Because of the small width of the $\omega$ meson and the narrow energy region of $\rho-\omega$ mixing, we fixed them. In fact, the errors of $m_{\omega}$ and $\Gamma_{\omega}$ only contributed tiny influences to the uncertainties of final results as found later. 
Another thing should be mentioned: the parameters $M_{\rho}$ and $\Gamma_{\rho}$ are model dependent. Thus, the differences between these models can be seen from our results in the next section; see more discussions later.

\section{RESULTS}

For the data of the PVFF below the energy region of $1 \mathrm{GeV}$, we take them from the experimental collaborations of Orsay, DM1, OLYA, CMD1, CMD2, BABAR, BESIII, KLOE, and SND [102-120]. Our fitting results for each set of experimental data are given in Table I, in which the details of $\chi^{2} /$ dof for the combined data (Com. Dat.) are given in the last line. In fact, there are two general parameters for all the models, $\alpha$ and $\kappa$ appearing in $P(s)$; see Eq. (54). For the Omnés model, there is no extra parameter. In the one of HL, there are three more, $a, b$, and $c$, as discussed above. Besides, the other BW types, including BW1, BW2, GS, and KS models, have another two parameters for the $\rho$ meson, $M_{\rho}$ and $\Gamma_{\rho}$. From the results of Table I, one can see that $\chi^{2} /$ dof for Orsay 1969 data are all too small due to only a few data points, see Fig. 1, and the ones for KLOE2005 are much larger than the others, owing to the fitted discrepancy around the $\rho-\omega$ mixing region. With the summarized results in Table I and a systematic analysis of all the fitting results, one can easily find that the results with the GS and KS models are better than the others, and are compatible with each other. Indeed, the Omnés model has only two free parameters, but it needs the $P$-wave phase shift as input, which is constrained by analyticity and unitarity, as well as crossing symmetry, and depends on the accuracy of the measured $\pi \pi$ $P$-wave phase shift. The HL model, in fact, used the $N / D$ method for the elastic $P$-wave $\pi \pi$ scattering amplitude with a simple one-pole contribution without considering the detail of the pole width, which can be matched with the simple BW pole ansatz; see the results later. For the BW1 model, it is only a simple BW pole ansatz from the vector meson dominance, which violates the charge normalization condition as discussed in the last section and is improved by the BW2 model with an energy-dependent decay width. Utilizing the unitarity condition and considering the detail of the energy dependence of the resonance width for the $\rho$ propagator, this is done in the GS and KS models. Therefore, it is not surprising that the fitting results of the GS and KS models are the best. Thus, our final results are favored with using the GS (or KS) model for the fit of the Com. Dat.. In Figs. 1 and 2, we show the results fitted with GS model for each set of experimental data and the Com. Dat., respectively. Note that some data above $1 \mathrm{GeV}$ in the sets of DM1-1978, OLYA1985, and BABAR have been ignored.

Furthermore, using the BW types of BW1, BW2, GS, and KS models, one can also determine the parameters of the $\rho$ meson, $M_{\rho}$ and $\Gamma_{\rho}$, from the fits; see the results of Table II. Note that in the first column the results for the HL model are not obtained directly from the fits. As discussed in Refs. [96,121], once the parameters $a, b$, and $c$ were determined from the fit, the $M_{\rho}$ and $\Gamma_{\rho}$ could also be determined, since the function $f(s)$, see Eq. (33), should be

TABLE I. Results of $\chi^{2} /$ dof for each fit in different sets of experimental data.

\begin{tabular}{|c|c|c|c|c|c|c|}
\hline Dataset & Omnés & HL & BW1 & BW2 & GS & $\mathrm{KS}$ \\
\hline $\begin{array}{l}\text { Orsay1969 [102] } \\
\text { [192 }\end{array}$ & 0.63 & $\ldots^{\mathrm{a}}$ & 0.02 & 0.02 & 0.01 & 0.01 \\
\hline DM1-1978 [103] & 0.74 & 1.99 & 0.96 & 0.92 & 0.81 & 0.81 \\
\hline OLYA1985 [104] & 0.57 & 0.59 & 0.54 & 0.54 & 0.58 & 0.58 \\
\hline CMD1985 [104] & 1.72 & 1.31 & 1.92 & 1.78 & 1.68 & 1.68 \\
\hline CMD2-2002 [105] & 1.14 & 1.32 & 1.11 & 1.11 & 1.14 & 1.14 \\
\hline CMD2-2004 [106] & 1.14 & 1.20 & 1.15 & 1.14 & 1.17 & 1.17 \\
\hline CMD2-2006 [108] & 1.43 & 12.72 & 1.7 & 1.72 & 1.77 & 1.79 \\
\hline CMD2-2007 [109] & 2.33 & 2.71 & 2.13 & 2.04 & 1.86 & 1.86 \\
\hline$B A B A R 2009$ [110] & 1.83 & 1.06 & 1.34 & 1.08 & 1.05 & 1.05 \\
\hline BESIII2020 [111,112] & 1.05 & 1.10 & 0.84 & 0.86 & 0.95 & 0.95 \\
\hline KLOE2005 [113] & 68.42 & 19.18 & 21.34 & 20.52 & 18.84 & 18.84 \\
\hline KLOE2009 [114] & 4.74 & 2.28 & 6.92 & 5.3 & 2.24 & 2.24 \\
\hline KLOE2011 [115] & 1.09 & 1.09 & 1.31 & 1.15 & 1.08 & 1.08 \\
\hline KLOE2013 [116] & 1.27 & 1.13 & 1.53 & 1.35 & 1.11 & 1.11 \\
\hline KLOE2018 [117] & 1.26 & 0.77 & 2.08 & 1.53 & 0.77 & 0.77 \\
\hline SND2005 [118] & 4.05 & 3.52 & 3.54 & 3.45 & 3.43 & 3.43 \\
\hline SND2006 [119] & 4.04 & 3.61 & 3.38 & 3.36 & 3.52 & 3.52 \\
\hline SND2020 [120] & 3.55 & 3.93 & 3.39 & 3.43 & 3.68 & 3.68 \\
\hline Com. Dat. & 11.29 & 10.20 & 11.01 & 10.65 & 10.19 & 10.19 \\
\hline$\frac{\chi^{2}}{\text { dof }}$ (Com. Dat.) & $\frac{11337.26}{1006-2}$ & $\frac{10214.46}{1006-5}$ & $\frac{11032.14}{1006-4}$ & $\frac{10671.72}{1006-4}$ & $\frac{10214.59}{1006-4}$ & $\frac{10214.59}{1006-4}$ \\
\hline
\end{tabular}

${ }^{\mathrm{a}}$ This is due to $d o f=0$ with only five data points available. 


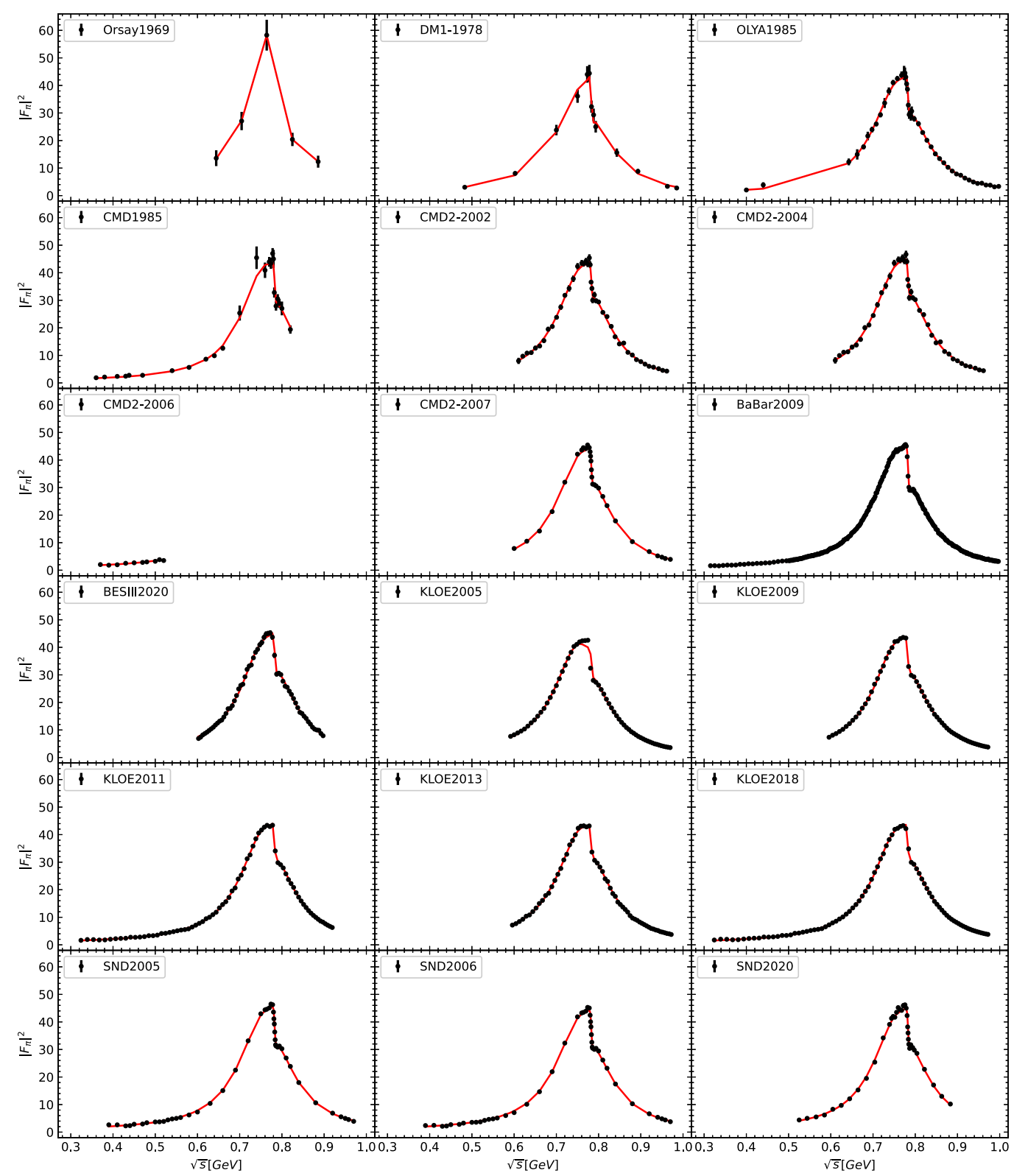

FIG. 1. Fitting results with the GS model for each set of experimental data.

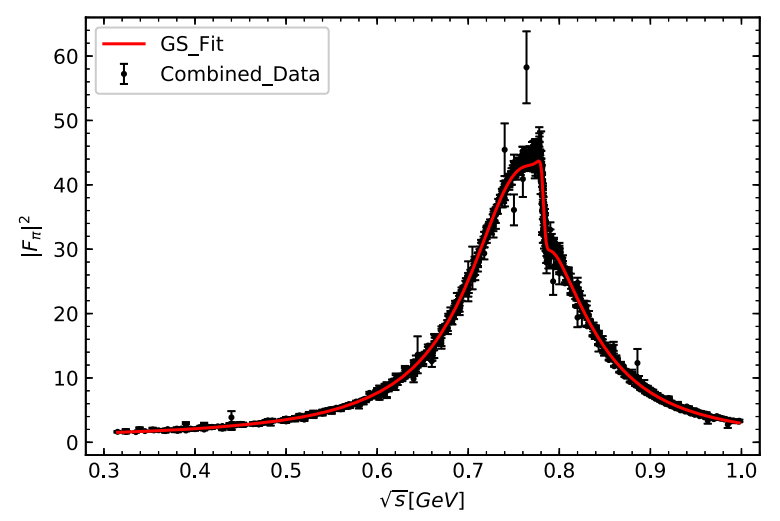

FIG. 2. Fitting results with the GS model for the Com. Dat.. matched with the BW form when $s \rightarrow M_{\rho}^{2}$. And thus, one can have $\operatorname{Re}\left[f\left(M_{\rho}^{2}\right)\right]=0$ and $\operatorname{Im}\left[f\left(M_{\rho}^{2}\right)\right]=-M_{\rho} \Gamma_{\rho}$. Since the small uncertainties are obtained from the fits for the parameters of $a, b$, and $c$, the uncertainties for the results of determining $M_{\rho}$ and $\Gamma_{\rho}$, and also for the results of $a_{\mu}^{\mathrm{HVP}, \mathrm{LO}}$ later, are small. For the data of CMD2-2006, because the data are close to the $\pi \pi$ threshold, it is not possible to get the results for $M_{\rho}$ and $\Gamma_{\rho}$ correctly. The results for Orsay 1969 are bigger than the others, whereas the ones for KLOE2005 are much smaller. And compared with different models, the results with the BW2 model are bigger than the values with the BW1 model and also larger than the numbers obtained with the GS (or KS) model. Finally, we obtain the results of the $\rho$ meson parameters, 
TABLE II. Results for the $\rho$ meson parameters $M_{\rho}$ and $\Gamma_{\rho}$ from the fits.

\begin{tabular}{|c|c|c|c|c|c|}
\hline Dataset & HL & BW1 & BW2 & GS & KS \\
\hline \multirow[t]{2}{*}{ Orsay 1969} & $779.00 \pm 0.00$ & $762.10 \pm 8.99$ & $793.08 \pm 13.13$ & $790.76 \pm 12.59$ & $790.72 \pm 12.59$ \\
\hline & $158.47 \pm 0.00$ & $178.58 \pm 32.87$ & $193.37 \pm 40.43$ & $190.88 \pm 38.85$ & $190.59 \pm 38.78$ \\
\hline \multirow[t]{2}{*}{ DM1-1978 } & $767.53 \pm 0.00$ & $757.41 \pm 3.52$ & $778.05 \pm 3.44$ & $776.83 \pm 3.49$ & $776.83 \pm 3.49$ \\
\hline & $155.10 \pm 0.00$ & $144.63 \pm 5.11$ & $153.03 \pm 6.01$ & $152.50 \pm 5.83$ & $152.50 \pm 5.83$ \\
\hline \multirow[t]{2}{*}{ OLYA1985 } & $775.81 \pm 5.75$ & $757.41 \pm 0.87$ & $777.54 \pm 0.77$ & $775.87 \pm 0.78$ & $775.87 \pm 0.78$ \\
\hline & $157.54 \pm 1.69$ & $143.43 \pm 1.32$ & $151.72 \pm 1.56$ & $151.71 \pm 1.53$ & $151.71 \pm 1.53$ \\
\hline \multirow[t]{2}{*}{ CMD1985 } & $761.57 \pm 102.54$ & $756.54 \pm 2.09$ & $774.29 \pm 3.33$ & $772.47 \pm 3.15$ & $772.47 \pm 3.14$ \\
\hline & $153.34 \pm 30.26$ & $134.54 \pm 6.51$ & $140.22 \pm 7.19$ & $136.14 \pm 6.75$ & $136.12 \pm 6.73$ \\
\hline \multirow[t]{2}{*}{ CMD2-2002 } & $775.38 \pm 0.00$ & $758.39 \pm 0.63$ & $777.83 \pm 0.62$ & $776.40 \pm 0.62$ & $776.40 \pm 0.62$ \\
\hline & $157.41 \pm 0.00$ & $140.96 \pm 1.22$ & $148.65 \pm 1.43$ & $148.17 \pm 1.39$ & $148.17 \pm 1.39$ \\
\hline \multirow[t]{2}{*}{ CMD2-2004 } & $776.02 \pm 4.92$ & $758.14 \pm 0.62$ & $777.53 \pm 0.61$ & $776.07 \pm 0.62$ & $776.07 \pm 0.62$ \\
\hline & $157.60 \pm 1.45$ & $140.78 \pm 1.22$ & $148.43 \pm 1.42$ & $147.90 \pm 1.38$ & $147.90 \pm 1.38$ \\
\hline CMD2-2006 & $\ldots$ & $\ldots$ & $\ldots$ & $\ldots$ & $\ldots$ \\
\hline \multirow{2}{*}{ CMD2-2007 } & $775.70 \pm 0.00$ & $757.82 \pm 0.44$ & $777.91 \pm 0.44$ & $776.35 \pm 0.44$ & $776.35 \pm 0.44$ \\
\hline & $157.50 \pm 0.00$ & $143.26 \pm 0.57$ & $151.46 \pm 0.68$ & $151.21 \pm 0.66$ & $151.21 \pm 0.66$ \\
\hline \multirow[t]{2}{*}{ BABAR2009 } & $774.53 \pm 0.00$ & $755.14 \pm 0.11$ & $775.85 \pm 0.11$ & $774.58 \pm 0.11$ & $774.58 \pm 0.11$ \\
\hline & $157.16 \pm 0.00$ & $144.60 \pm 0.24$ & $152.70 \pm 0.28$ & $151.38 \pm 0.27$ & $151.38 \pm 0.27$ \\
\hline \multirow[t]{2}{*}{ BESIII2020 } & $775.86 \pm 0.00$ & $757.87 \pm 0.36$ & $777.73 \pm 0.40$ & $776.22 \pm 0.39$ & $776.22 \pm 0.39$ \\
\hline & $157.55 \pm 0.00$ & $142.52 \pm 1.05$ & $149.93 \pm 1.21$ & $147.87 \pm 1.17$ & $147.87 \pm 1.17$ \\
\hline \multirow[t]{2}{*}{ KLOE2005 } & $768.79 \pm 2.09$ & $750.52 \pm 0.13$ & $770.43 \pm 0.12$ & $768.87 \pm 0.12$ & $768.87 \pm 0.12$ \\
\hline & $155.47 \pm 0.62$ & $141.37 \pm 0.19$ & $149.63 \pm 0.22$ & $149.67 \pm 0.22$ & $149.67 \pm 0.22$ \\
\hline \multirow[t]{2}{*}{ KLOE2009 } & $774.86 \pm 2.06$ & $756.58 \pm 0.09$ & $776.41 \pm 0.08$ & $774.93 \pm 0.08$ & $774.93 \pm 0.08$ \\
\hline & $157.26 \pm 0.61$ & $142.07 \pm 0.13$ & $150.17 \pm 0.15$ & $150.18 \pm 0.14$ & $150.18 \pm 0.14$ \\
\hline \multirow[t]{2}{*}{ KLOE2011 } & $775.61 \pm 0.00$ & $757.25 \pm 0.19$ & $777.11 \pm 0.16$ & $775.66 \pm 0.17$ & $775.66 \pm 0.17$ \\
\hline & $157.48 \pm 0.00$ & $142.24 \pm 0.32$ & $150.09 \pm 0.38$ & $149.29 \pm 0.37$ & $149.29 \pm 0.37$ \\
\hline \multirow[t]{2}{*}{ KLOE2013 } & $775.69 \pm 2.38$ & $757.30 \pm 0.28$ & $776.99 \pm 0.26$ & $775.76 \pm 0.27$ & $775.76 \pm 0.27$ \\
\hline & $157.50 \pm 0.70$ & $141.34 \pm 0.41$ & $149.40 \pm 0.49$ & $149.86 \pm 0.48$ & $149.86 \pm 0.48$ \\
\hline \multirow[t]{2}{*}{ KLOE2018 } & $775.15 \pm 2.67$ & $756.63 \pm 0.14$ & $776.57 \pm 0.12$ & $775.22 \pm 0.13$ & $775.22 \pm 0.13$ \\
\hline & $157.34 \pm 0.78$ & $142.29 \pm 0.20$ & $150.37 \pm 0.24$ & $150.21 \pm 0.23$ & $150.21 \pm 0.23$ \\
\hline \multirow[t]{2}{*}{ SND2005 } & $775.27 \pm 0.00$ & $756.46 \pm 0.26$ & $776.92 \pm 0.27$ & $775.33 \pm 0.27$ & $775.33 \pm 0.27$ \\
\hline & $157.38 \pm 0.00$ & $144.34 \pm 0.49$ & $152.62 \pm 0.58$ & $151.88 \pm 0.56$ & $151.88 \pm 0.56$ \\
\hline \multirow[t]{2}{*}{ SND2006 } & $775.54 \pm 1.92$ & $756.70 \pm 0.27$ & $777.14 \pm 0.27$ & $775.60 \pm 0.27$ & $775.60 \pm 0.27$ \\
\hline & $157.46 \pm 0.56$ & $144.27 \pm 0.49$ & $152.55 \pm 0.57$ & $151.91 \pm 0.56$ & $151.91 \pm 0.56$ \\
\hline \multirow[t]{2}{*}{ SND2020 } & $775.59 \pm 0.00$ & $757.33 \pm 0.29$ & $777.67 \pm 0.33$ & $775.92 \pm 0.32$ & $775.92 \pm 0.32$ \\
\hline & $157.47 \pm 0.00$ & $144.33 \pm 0.81$ & $152.08 \pm 0.94$ & $149.98 \pm 0.90$ & $149.98 \pm 0.90$ \\
\hline \multirow[t]{2}{*}{ Com. Dat. } & $774.00 \pm 0.79$ & $755.62 \pm 0.05$ & $775.44 \pm 0.04$ & $774.07 \pm 0.04$ & $774.07 \pm 0.04$ \\
\hline & $157.00 \pm 0.23$ & $141.74 \pm 0.07$ & $149.76 \pm 0.08$ & $149.54 \pm 0.08$ & $149.54 \pm 0.08$ \\
\hline
\end{tabular}

$M_{\rho}$ and $\Gamma_{\rho}{ }^{3}$ from the fit with GS (or KS) model and the Com. Dat.,

$M_{\rho}=(774.07 \pm 0.04) \mathrm{MeV}, \quad \Gamma_{\rho}=(149.54 \pm 0.08) \mathrm{MeV}$

which are $1 \mathrm{MeV}$ smaller than the result reported by the PDG [34], (775.26 \pm 0.23$) \mathrm{MeV}$, for the mass, and $2 \mathrm{MeV}$ bigger than the value $(147.4 \pm 0.8) \mathrm{MeV}$ for the width and a bit smaller than the other result, $M_{\rho}=(775.13 \pm 0.02) \mathrm{MeV}$, obtained in Ref. [86]. Our results are also consistent with the value obtained in Ref. [12], $M_{\rho}=(774.5 \pm 0.8) \mathrm{MeV}$.

\footnotetext{
${ }^{3}$ In fact, these results are for the neutral one of the $\rho^{0}$ meson in the $e^{+} e^{-}$annihilation processes.
}

Note that the small errors for the pole parameters are due to more constraints in the Com. Dat.. We should remark that, as discussed above and shown in Table II, the $\rho$ meson parameters, $M_{\rho}$ and $\Gamma_{\rho}$, are model dependent and do not correspond to the physical resonance's mass and width, which should be determined by looking for the pole in the second Riemann sheet. It is complicated to extrapolate the form factor to the second Riemann sheet [122], which is out of our concern in the present work but for which one can refer to Refs. $[69,123]$ for more discussions ${ }^{4}$ and Ref. [124] for the application in the unitarized three-body BW function.

\footnotetext{
${ }^{4}$ In their model, a pole $\left(762.0 \pm 0.3, \frac{i}{2}(143.0 \pm 0.2)\right) \mathrm{MeV}$ was found for a mass parameter $M_{\rho}=(775.2 \pm 0.4) \mathrm{MeV}$ from the fitting of $\tau$ decay data.
} 
TABLE III. Results of $a_{\mu}^{\mathrm{HVP}, \mathrm{LO}}\left(\pi^{+} \pi^{-}\right)\left(\times 10^{-10}\right)$ from two-pion contribution up to $1 \mathrm{GeV}$.

\begin{tabular}{lcccccl}
\hline \hline Dataset & Omnés & HL & BW1 & BW2 & GS & KS \\
\hline Orsay1969 & $555.48 \pm 29.98$ & $553.24 \pm 0.16$ & $562.66 \pm 174.98$ & $565.56 \pm 165.17$ & $568.00 \pm 139.17$ & $567.72 \pm 138.87$ \\
DM1-1978 & $468.24 \pm 8.97$ & $491.35 \pm 0.01$ & $463.46 \pm 30.03$ & $465.12 \pm 29.31$ & $467.90 \pm 25.56$ & $467.90 \pm 25.59$ \\
OLYA1985 & $490.73 \pm 2.32$ & $490.64 \pm 9.98$ & $483.72 \pm 7.80$ & $486.07 \pm 7.68$ & $490.64 \pm 6.68$ & $490.64 \pm 6.69$ \\
CMD1985 & $475.48 \pm 6.12$ & $461.50 \pm 76.32$ & $470.32 \pm 34.68$ & $469.71 \pm 32.50$ & $467.19 \pm 27.65$ & $467.20 \pm 27.64$ \\
CMD2-2002 & $492.46 \pm 2.06$ & $495.36 \pm 0.01$ & $483.93 \pm 7.31$ & $485.80 \pm 7.15$ & $489.02 \pm 6.24$ & $489.02 \pm 6.23$ \\
CMD2-2004 & $503.86 \pm 2.11$ & $501.27 \pm 9.02$ & $496.16 \pm 7.49$ & $498.06 \pm 7.33$ & $501.26 \pm 6.40$ & $501.26 \pm 6.41$ \\
CMD2-2006 & $\ldots$ & $\ldots$ & $\ldots$ & $\ldots$ & $\ldots$ & $\ldots$ \\
CMD2-2007 & $501.45 \pm 0.99$ & $501.58 \pm 0.01$ & $494.63 \pm 3.86$ & $496.76 \pm 3.80$ & $500.57 \pm 3.36$ & $500.57 \pm 3.36$ \\
BABAR2009 & $501.38 \pm 0.41$ & $503.35 \pm 0.01$ & $500.01 \pm 1.28$ & $501.45 \pm 1.24$ & $503.36 \pm 1.06$ & $503.36 \pm 1.06$ \\
BESIII2020 & $498.39 \pm 1.56$ & $500.35 \pm 0.01$ & $493.96 \pm 5.82$ & $495.35 \pm 5.63$ & $496.93 \pm 4.83$ & $496.93 \pm 4.83$ \\
KLOE2005 & $473.67 \pm 0.32$ & $496.27 \pm 7.30$ & $490.14 \pm 1.08$ & $492.27 \pm 1.06$ & $496.25 \pm 0.92$ & $496.25 \pm 0.92$ \\
KLOE2009 & $495.06 \pm 0.22$ & $497.11 \pm 7.44$ & $491.06 \pm 0.72$ & $493.19 \pm 0.71$ & $497.09 \pm 0.62$ & $497.09 \pm 0.61$ \\
KLOE2011 & $494.60 \pm 0.52$ & $493.81 \pm 0.01$ & $489.13 \pm 1.89$ & $490.92 \pm 1.85$ & $493.77 \pm 1.59$ & $493.77 \pm 1.59$ \\
KLOE2013 & $495.90 \pm 0.62$ & $495.57 \pm 6.02$ & $490.64 \pm 2.10$ & $492.45 \pm 2.09$ & $495.56 \pm 1.80$ & $495.56 \pm 1.80$ \\
KLOE2018 & $495.21 \pm 0.35$ & $495.95 \pm 7.13$ & $490.53 \pm 1.13$ & $492.49 \pm 1.12$ & $495.93 \pm 0.96$ & $495.93 \pm 0.96$ \\
SND2005 & $508.68 \pm 0.77$ & $513.28 \pm 0.01$ & $508.34 \pm 3.19$ & $510.25 \pm 3.12$ & $513.28 \pm 2.73$ & $513.28 \pm 2.75$ \\
SND2006 & $497.75 \pm 0.76$ & $501.18 \pm 3.61$ & $496.16 \pm 3.07$ & $498.05 \pm 3.01$ & $501.17 \pm 2.63$ & $501.17 \pm 2.65$ \\
SND2020 & $500.47 \pm 0.99$ & $499.04 \pm 0.01$ & $498.29 \pm 5.04$ & $499.63 \pm 4.89$ & $501.04 \pm 4.27$ & $501.04 \pm 4.29$ \\
Com. Dat. & $492.81 \pm 1.00$ & $497.78 \pm 3.61$ & $492.57 \pm 3.70$ & $494.46 \pm 3.62$ & $497.76 \pm 3.15$ & $497.76 \pm 3.15$ \\
\hline \hline
\end{tabular}

Besides, for the other parameters, we show the results in Table IV in the Appendix, in which the details for $\alpha$ and $\kappa$ are given and the results with Omnés model are consistent with the results of Ref. [100] within the uncertainties. As done in Ref. [100], one can extract the $\omega \pi \pi$ coupling and the branching ratio of $\omega \rightarrow \pi \pi$ from the results of $\kappa$ parameter in Table IV. Thus, following the same way and using the favored results of the GS (or KS) model for the Com. Dat., $\kappa=(1.77 \pm 0.01) \times 10^{-3}$, we have

$$
\begin{aligned}
g_{\omega \pi \pi} & =(3.02 \pm 0.05) \times 10^{-2}, \\
\operatorname{Br}(\omega \rightarrow \pi \pi) & =(1.52 \pm 0.06) \%,
\end{aligned}
$$

which are consistent with the results obtained in Ref. [100] within the uncertainties. Our result for the branching ratio is also in good agreement with that of the PDG [34], $\operatorname{Br}(\omega \rightarrow \pi \pi)=\left(1.53_{-0.13}^{+0.11}\right) \%$.

With the fitting results obtained above with different models of the PVFF for the data below $1 \mathrm{GeV}$, we can evaluate the value of $a_{\mu}^{\mathrm{HVP}, \mathrm{LO}}\left(\pi^{+} \pi^{-}\right)$from the two-pion contribution with the dispersion integral defined in Eq. (22). Our results are given in Table III, using different PVFF models for each set of experimental data up to $1 \mathrm{GeV}$, except for the data of CMD2-2006. We also show the results with GS model for the Com. Dat. in Fig. 3 clearly. As one can find in Table III, the results for Orsay1969 are bigger than the others, and conversely, the results for CMD1985 are smaller than the others. The uncertainties for the results of Omnés model are smaller than the others, whereas the results with the BW1 model are the biggest; see Fig. 3. Indeed, from Fig. 3, compared to the results with GS or KS model, there is a $1 \%$ difference from the smallest value with the BW1 model. At the end, our final results are taken from the GS (or KS) model using the Com. Dat., given as

$$
a_{\mu}^{\mathrm{HVP}, \mathrm{LO}}\left(\pi^{+} \pi^{-} \leq 1 \mathrm{GeV}\right)=(497.76 \pm 3.15) \times 10^{-10},
$$

which are very consistent with the result obtained in Ref. [78], $a_{\mu}^{\mathrm{HVP}}(\pi \pi \leq 1 \mathrm{GeV})=(497.0 \pm 1.4) \times 10^{-10}$, an updated result of Ref. [64] by considering the inelastic effects from the constraints of the Eidelman-Łukaszuk bound. Furthermore, using the framework of resonance

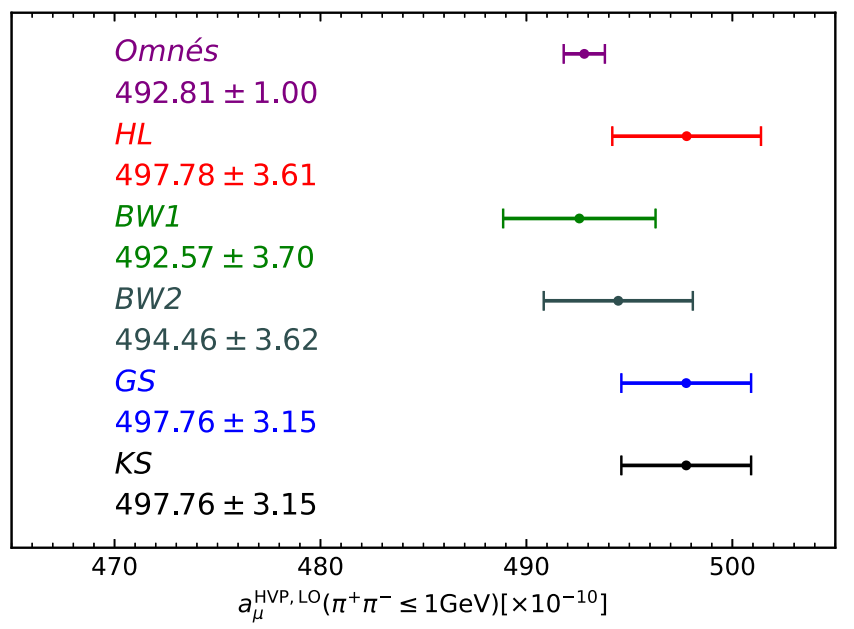

FIG. 3. Summarized results of $a_{\mu}^{\mathrm{HVP}, \mathrm{LO}}\left(\pi^{+} \pi^{-}\right)$from two-pion contribution up to $1 \mathrm{GeV}$ using the GS model and fitting with the Com. Dat.. 
chiral theory, two similar values of $a_{\mu}^{\mathrm{HVP}}(\pi \pi \leq 1 \mathrm{GeV})=$ $(498.48 \pm 2.34) \times 10^{-10}$ (fit I) and $(498.47 \pm 2.33) \times 10^{-10}$ (fit II) were obtained in Ref. [125] and are consistent with ours within the uncertainties. One thing should be mentioned: the obtained error is estimated by the average of the reasonable errors in Table III for different sets of data, since one can find that the errors for different sets of data are mainly contributed by the errors of the pole parameters, see Table II, and the errors for four sets of data are quite large due to the fewer data points in the $\rho$ region. This is why the error is so small for the result of the Omnés model with no pole parameter; see the results of Table III.

\section{CONCLUSION}

In the present work, in order to reduce the uncertainties of the calculation of two-pion contribution to the muon anomalous magnetic moment, we try to get the best fit for the two-pion cross sections with several theoretical models of the PVFF, combined with a polynomial description. Since the polynomial description is valid up to $1 \mathrm{GeV}$, we only take into account all the experimental data below $1 \mathrm{GeV}$, which is below the significant inelastic threshold and contributes almost more than $70 \%$ of the hadronic contribution to the muon anomalous magnetic moment. From our results, we find that the fit with the GounarisSakurai (or Kühn-Santamaria) model is the best one. From the best fit to the PVFF, one can also extract the branching ratio of $\omega \rightarrow \pi \pi$, given by

$$
\operatorname{Br}(\omega \rightarrow \pi \pi)=(1.52 \pm 0.06) \%,
$$

which is compatible with the results reported by the Particle Data Group. Based on the best fit to data, we calculate the two-pion contribution to the muon anomalous magnetic moment, obtaining

$$
a_{\mu}^{\mathrm{HVP}, \mathrm{LO}}\left(\pi^{+} \pi^{-} \leq 1 \mathrm{GeV}\right)=(497.76 \pm 3.15) \times 10^{-10},
$$

which is in good agreement with the recent theoretical evaluations $[78,125]$. Our results for two-pion contribution are helpful to pin down the uncertainties of the calculation for the hadronic vacuum polarization contribution to the muon anomalous magnetic moment.

\section{ACKNOWLEDGMENTS}

We thank Professors Martin Hoferichter, Irinel Caprini, Hidezumi Terazawa, Peter Athron, Yusi Pan, Wen Yin, and Nikolay N. Achasov for valuable comments and useful information and acknowledge the referee for helpful suggestions.

\section{APPENDIX: THE OTHER PARAMETERS}

We give the details of some other parameters in Table IV, in which the values of $\alpha$ and $\kappa$ are shown for each fit with different PVFF models and the parameters with Omnés model are consistent with the results obtained in Ref. [100] within the uncertainties. 
TABLE IV. Parameters of $\alpha\left(\mathrm{GeV}^{-2}\right)$ and $\kappa \times 10^{3}$ for each fit.

\begin{tabular}{|c|c|c|c|c|c|c|}
\hline Dataset & Omnés & $\mathrm{HL}$ & BW1 & BW2 & GS & KS \\
\hline \multirow[t]{2}{*}{ Orsay 1969} & $0.15 \pm 0.05$ & $0.23 \pm 0.00$ & $0.73 \pm 0.32$ & $0.69 \pm 0.29$ & $0.45 \pm 0.22$ & $0.45 \pm 0.22$ \\
\hline & $7.68 \pm 2.75$ & $9.26 \pm 0.00$ & $18.72 \pm 8.63$ & $18.52 \pm 8.33$ & $16.79 \pm 7.21$ & $16.74 \pm 7.20$ \\
\hline \multirow[t]{2}{*}{ DM1-1978 } & $0.01 \pm 0.02$ & $0.12 \pm 0.00$ & $0.30 \pm 0.06$ & $0.28 \pm 0.06$ & $0.12 \pm 0.05$ & $0.12 \pm 0.05$ \\
\hline & $2.14 \pm 0.47$ & $1.64 \pm 0.00$ & $2.61 \pm 0.61$ & $2.59 \pm 0.60$ & $2.41 \pm 0.55$ & $2.41 \pm 0.55$ \\
\hline \multirow[t]{2}{*}{ OLYA1985 } & $0.06 \pm 0.00$ & $0.16 \pm 0.01$ & $0.34 \pm 0.02$ & $0.32 \pm 0.01$ & $0.17 \pm 0.01$ & $0.17 \pm 0.01$ \\
\hline & $1.68 \pm 0.17$ & $1.93 \pm 0.21$ & $2.06 \pm 0.23$ & $2.05 \pm 0.23$ & $1.94 \pm 0.21$ & $1.94 \pm 0.21$ \\
\hline \multirow[t]{2}{*}{ CMD1985 } & $0.02 \pm 0.01$ & $-0.07 \pm 0.05$ & $0.23 \pm 0.06$ & $0.20 \pm 0.06$ & $0.02 \pm 0.05$ & $0.02 \pm 0.05$ \\
\hline & $2.03 \pm 0.26$ & $1.52 \pm 0.30$ & $2.05 \pm 0.38$ & $2.02 \pm 0.37$ & $1.79 \pm 0.33$ & $1.79 \pm 0.33$ \\
\hline \multirow[t]{2}{*}{ CMD2-2002 } & $0.06 \pm 0.00$ & $0.15 \pm 0.00$ & $0.32 \pm 0.01$ & $0.30 \pm 0.01$ & $0.14 \pm 0.01$ & $0.14 \pm 0.01$ \\
\hline & $1.48 \pm 0.13$ & $1.50 \pm 0.00$ & $1.72 \pm 0.16$ & $1.72 \pm 0.16$ & $1.62 \pm 0.15$ & $1.62 \pm 0.15$ \\
\hline \multirow[t]{2}{*}{ CMD2-2004 } & $0.08 \pm 0.00$ & $0.16 \pm 0.01$ & $0.35 \pm 0.01$ & $0.33 \pm 0.01$ & $0.17 \pm 0.01$ & $0.17 \pm 0.01$ \\
\hline & $1.50 \pm 0.13$ & $1.60 \pm 0.15$ & $1.71 \pm 0.16$ & $1.71 \pm 0.16$ & $1.60 \pm 0.15$ & $1.60 \pm 0.15$ \\
\hline CMD2-2006 & $\ldots$ & $\ldots$ & $\ldots$ & $\ldots$ & $\ldots$ & \\
\hline \multirow[t]{2}{*}{ CMD2-2007 } & $0.08 \pm 0.00$ & $0.17 \pm 0.00$ & $0.37 \pm 0.01$ & $0.35 \pm 0.01$ & $0.19 \pm 0.01$ & $0.19 \pm 0.01$ \\
\hline & $1.54 \pm 0.05$ & $1.61 \pm 0.00$ & $1.87 \pm 0.07$ & $1.86 \pm 0.07$ & $1.74 \pm 0.06$ & $1.74 \pm 0.06$ \\
\hline \multirow[t]{2}{*}{$B A B A R 2009$} & $0.08 \pm 0.00$ & $0.19 \pm 0.00$ & $0.39 \pm 0.00$ & $0.37 \pm 0.00$ & $0.19 \pm 0.00$ & $0.19 \pm 0.00$ \\
\hline & $2.16 \pm 0.03$ & $2.25 \pm 0.00$ & $2.39 \pm 0.05$ & $2.39 \pm 0.05$ & $2.26 \pm 0.04$ & $2.26 \pm 0.04$ \\
\hline \multirow[t]{2}{*}{ BESIII2020 } & $0.07 \pm 0.00$ & $0.16 \pm 0.00$ & $0.36 \pm 0.01$ & $0.33 \pm 0.01$ & $0.16 \pm 0.01$ & $0.16 \pm 0.01$ \\
\hline & $1.60 \pm 0.13$ & $1.60 \pm 0.00$ & $1.98 \pm 0.18$ & $1.96 \pm 0.17$ & $1.82 \pm 0.16$ & $1.82 \pm 0.16$ \\
\hline \multirow[t]{2}{*}{ KLOE2005 } & $0.02 \pm 0.00$ & $0.16 \pm 0.00$ & $0.35 \pm 0.00$ & $0.33 \pm 0.00$ & $0.17 \pm 0.00$ & $0.17 \pm 0.00$ \\
\hline & $1.78 \pm 0.03$ & $1.31 \pm 0.04$ & $1.36 \pm 0.04$ & $1.37 \pm 0.04$ & $1.32 \pm 0.04$ & $1.32 \pm 0.04$ \\
\hline \multirow[t]{2}{*}{ KLOE2009 } & $0.07 \pm 0.00$ & $0.17 \pm 0.00$ & $0.35 \pm 0.00$ & $0.33 \pm 0.00$ & $0.17 \pm 0.00$ & $0.17 \pm 0.00$ \\
\hline & $1.66 \pm 0.02$ & $1.75 \pm 0.03$ & $1.81 \pm 0.03$ & $1.82 \pm 0.03$ & $1.75 \pm 0.03$ & $1.75 \pm 0.03$ \\
\hline \multirow[t]{2}{*}{ KLOE2011 } & $0.06 \pm 0.00$ & $0.15 \pm 0.00$ & $0.35 \pm 0.00$ & $0.32 \pm 0.00$ & $0.16 \pm 0.00$ & $0.16 \pm 0.00$ \\
\hline & $1.57 \pm 0.05$ & $1.73 \pm 0.00$ & $1.82 \pm 0.06$ & $1.83 \pm 0.06$ & $1.74 \pm 0.06$ & $1.74 \pm 0.06$ \\
\hline \multirow[t]{2}{*}{ KLOE2013 } & $0.07 \pm 0.00$ & $0.16 \pm 0.00$ & $0.34 \pm 0.00$ & $0.32 \pm 0.00$ & $0.17 \pm 0.00$ & $0.17 \pm 0.00$ \\
\hline & $1.39 \pm 0.10$ & $1.58 \pm 0.11$ & $1.57 \pm 0.12$ & $1.59 \pm 0.12$ & $1.59 \pm 0.11$ & $1.59 \pm 0.11$ \\
\hline \multirow[t]{2}{*}{ KLOE2018 } & $0.07 \pm 0.00$ & $0.16 \pm 0.00$ & $0.35 \pm 0.00$ & $0.33 \pm 0.00$ & $0.17 \pm 0.00$ & $0.17 \pm 0.00$ \\
\hline & $1.58 \pm 0.04$ & $1.69 \pm 0.05$ & $1.70 \pm 0.05$ & $1.72 \pm 0.05$ & $1.69 \pm 0.05$ & $1.69 \pm 0.05$ \\
\hline \multirow[t]{2}{*}{ SND2005 } & $0.09 \pm 0.00$ & $0.21 \pm 0.00$ & $0.41 \pm 0.01$ & $0.39 \pm 0.01$ & $0.22 \pm 0.01$ & $0.22 \pm 0.01$ \\
\hline & $1.71 \pm 0.04$ & $1.89 \pm 0.00$ & $2.06 \pm 0.05$ & $2.05 \pm 0.05$ & $1.90 \pm 0.04$ & $1.90 \pm 0.04$ \\
\hline \multirow[t]{2}{*}{ SND2006 } & $0.07 \pm 0.00$ & $0.19 \pm 0.00$ & $0.38 \pm 0.01$ & $0.36 \pm 0.01$ & $0.19 \pm 0.00$ & $0.19 \pm 0.00$ \\
\hline & $1.68 \pm 0.04$ & $1.87 \pm 0.04$ & $2.02 \pm 0.05$ & $2.01 \pm 0.05$ & $1.87 \pm 0.04$ & $1.87 \pm 0.04$ \\
\hline \multirow[t]{2}{*}{ SND2020 } & $0.08 \pm 0.00$ & $0.16 \pm 0.00$ & $0.39 \pm 0.01$ & $0.36 \pm 0.01$ & $0.18 \pm 0.01$ & $0.18 \pm 0.01$ \\
\hline & $1.74 \pm 0.05$ & $1.86 \pm 0.00$ & $2.11 \pm 0.07$ & $2.08 \pm 0.07$ & $1.90 \pm 0.06$ & $1.90 \pm 0.06$ \\
\hline \multirow{2}{*}{ Com. Dat. } & $0.06 \pm 0.00$ & $0.16 \pm 0.00$ & $0.35 \pm 0.00$ & $0.33 \pm 0.00$ & $0.17 \pm 0.00$ & $0.17 \pm 0.00$ \\
\hline & $1.76 \pm 0.01$ & $1.76 \pm 0.01$ & $1.84 \pm 0.01$ & $1.85 \pm 0.01$ & $1.77 \pm 0.01$ & $1.77 \pm 0.01$ \\
\hline
\end{tabular}

[1] R. L. Garwin, L. M. Lederman, and M. Weinrich, Phys. Rev. 105, 1415 (1957).

[2] R. L. Garwin, D. P. Hutchinson, S. Penman, and G. Shapiro, Phys. Rev. 118, 271 (1960).

[3] H. Terazawa, Prog. Theor. Phys. 39, 1326 (1968).

[4] H. Terazawa, Prog. Theor. Phys. 40, 830 (1968).

[5] H. Terazawa, Phys. Rev. 177, 2159 (1969).

[6] T. Kinoshita, J. Pestieau, P. Roy, and H. Terazawa, Phys. Rev. D 2, 910 (1970).
[7] H. Terazawa, Nonlin. Phenom. Complex Syst. 21, 268 (2018), http://www.j-npcs.org/abstracts/vol2018/v21no3/ v21no3p268.html.

[8] F. Jegerlehner and A. Nyffeler, Phys. Rep. 477, 1 (2009).

[9] J. P. Miller, E. de Rafael, B. L. Roberts, and D. Stöckinger, Annu. Rev. Nucl. Part. Sci. 62, 237 (2012).

[10] G. W. Bennett et al. (Muon g-2 Collaboration), Phys. Rev. D 73, 072003 (2006). 
[11] P. J. Mohr, B. N. Taylor, and D. B. Newell, Rev. Mod. Phys. 84, 1527 (2012).

[12] M. Davier, A. Hoecker, B. Malaescu, and Z. Zhang, Eur. Phys. J. C 80, 241 (2020); 80, 410(E) (2020).

[13] B. Abi et al. (Muon g-2 Collaboration), Phys. Rev. Lett. 126, 141801 (2021).

[14] T. Aoyama, N. Asmussen, M. Benayoun, J. Bijnens, T. Blum, M. Bruno, I. Caprini, C. M. Carloni Calame, M. Cè, and G. Colangelo et al., Phys. Rep. 887, 1 (2020).

[15] C. W. Chiang and K. Yagyu, Phys. Rev. D 103, L111302 (2021).

[16] A. E. Cárcamo Hernández, C. Espinoza, J. Carlos GómezIzquierdo, and M. Mondragón, arXiv:2104.02730.

[17] G. Arcadi, L. Calibbi, M. Fedele, and F. Mescia, Phys. Rev. Lett. 127, 061802 (2021).

[18] B. Zhu and X. Liu, arXiv:2104.03238.

[19] M. Endo, K. Hamaguchi, S. Iwamoto, and T. Kitahara, J. High Energy Phys. 07 (2021) 075.

[20] X. F. Han, T. Li, H. X. Wang, L. Wang, and Y. Zhang, arXiv:2104.03227.

[21] P. Das, M. K. Das, and N. Khan, arXiv:2104.03271.

[22] S. F. Ge, X. D. Ma, and P. Pasquini, Eur. Phys. J. C 81, 787 (2021).

[23] S. Baum, M. Carena, N. R. Shah, and C. E. M. Wagner, arXiv:2104.03302.

[24] H. B. Zhang, C. X. Liu, J. L. Yang, and T. F. Feng, arXiv:2104.03489.

[25] W. Ahmed, I. Khan, J. Li, T. Li, S. Raza, and W. Zhang, arXiv:2104.03491.

[26] J. Cao, J. Lian, Y. Pan, D. Zhang, and P. Zhu, J. High Energy Phys. 09 (2021) 175.

[27] A. Crivellin and M. Hoferichter, J. High Energy Phys. 07 (2021) 135.

[28] P. Athron, C. Balázs, D. H. Jacob, W. Kotlarski, D. Stöckinger, and H. Stöckinger-Kim, J. High Energy Phys. 09 (2021) 080.

[29] W. Yin and M. Yamaguchi, arXiv:2012.03928.

[30] W. Yin and W. Yin, J. High Energy Phys. 08 (2021) 043.

[31] W. Yin, J. High Energy Phys. 06 (2021) 029.

[32] H. Terazawa, Quark Matter: From Subquarks to the Universe, Physics Research and Technology (NOVA, Hauppauge, NY, 2018).

[33] A. Keshavarzi, K. S. Khaw, and T. Yoshioka, arXiv:2106. 06723.

[34] P. A. Zyla et al. (Particle Data Group), Prog. Theor. Exp. Phys. 2020, 083C01 (2020).

[35] T. Aoyama, M. Hayakawa, T. Kinoshita, and M. Nio, Phys. Rev. Lett. 109, 111807 (2012).

[36] T. Aoyama, M. Hayakawa, T. Kinoshita, and M. Nio, Phys. Rev. Lett. 109, 111808 (2012).

[37] P. A. Baikov, A. Maier, and P. Marquard, Nucl. Phys. B877, 647 (2013).

[38] S. Volkov, Phys. Rev. D 100, 096004 (2019).

[39] A. Czarnecki, W. J. Marciano, and A. Vainshtein, Phys. Rev. D 67, 073006 (2003); 73, 119901(E) (2006).

[40] C. Gnendiger, D. Stöckinger, and H. Stöckinger-Kim, Phys. Rev. D 88, 053005 (2013).

[41] T. Ishikawa, N. Nakazawa, and Y. Yasui, Phys. Rev. D 99, 073004 (2019).
[42] K. Melnikov and A. Vainshtein, Phys. Rev. D 70, 113006 (2004).

[43] P. Masjuan and P. Sánchez-Puertas, Phys. Rev. D 95, 054026 (2017).

[44] G. Colangelo, M. Hoferichter, M. Procura, and P. Stoffer, J. High Energy Phys. 04 (2017) 161.

[45] M. Hoferichter, B. L. Hoid, B. Kubis, S. Leupold, and S. P. Schneider, J. High Energy Phys. 10 (2018) 141.

[46] A. Gérardin, H. B. Meyer, and A. Nyffeler, Phys. Rev. D 100, 034520 (2019).

[47] J. Bijnens, N. Hermansson-Truedsson, and A. RodríguezSánchez, Phys. Lett. B 798, 134994 (2019).

[48] T. Blum, N. Christ, M. Hayakawa, T. Izubuchi, L. Jin, C. Jung, and C. Lehner, Phys. Rev. Lett. 124, 132002 (2020).

[49] E. H. Chao, R. J. Hudspith, A. Gérardin, J. R. Green, H. B. Meyer, and K. Ottnad, Eur. Phys. J. C 81, 651 (2021).

[50] J. Lüdtke and M. Procura, Eur. Phys. J. C 80, 1108 (2020).

[51] J. Bijnens, N. Hermansson-Truedsson, L. Laub, and A. Rodríguez-Sánchez, J. High Energy Phys. 10 (2020) 203.

[52] J. Bijnens, N. Hermansson-Truedsson, L. Laub, and A. Rodríguez-Sánchez, J. High Energy Phys. 04 (2021) 240.

[53] P. Masjuan, P. Roig, and P. Sanchez-Puertas, arXiv: 2005.11761.

[54] P. Roig and P. Sanchez-Puertas, Phys. Rev. D 101, 074019 (2020).

[55] L. Cappiello, O. Catà, G. D’Ambrosio, D. Greynat, and A. Iyer, Phys. Rev. D 102, 016009 (2020).

[56] M. Zanke, M. Hoferichter, and B. Kubis, J. High Energy Phys. 07 (2021) 106.

[57] J. Leutgeb and A. Rebhan, Phys. Rev. D 101, 114015 (2020).

[58] I. Danilkin, M. Hoferichter, and P. Stoffer, Phys. Lett. B 820, 136502 (2021).

[59] G. Colangelo, F. Hagelstein, M. Hoferichter, L. Laub, and P. Stoffer, Eur. Phys. J. C 81, 702 (2021).

[60] A. Keshavarzi, D. Nomura, and T. Teubner, Phys. Rev. D 101, 014029 (2020).

[61] A. Kurz, T. Liu, P. Marquard, and M. Steinhauser, Phys. Lett. B 734, 144 (2014).

[62] M. Davier, A. Hoecker, B. Malaescu, and Z. Zhang, Eur. Phys. J. C 77, 827 (2017).

[63] A. Keshavarzi, D. Nomura, and T. Teubner, Phys. Rev. D 97, 114025 (2018).

[64] G. Colangelo, M. Hoferichter, and P. Stoffer, J. High Energy Phys. 02 (2019) 006.

[65] M. Hoferichter, B. L. Hoid, and B. Kubis, J. High Energy Phys. 08 (2019) 137.

[66] S. Borsanyi, Z. Fodor, J. N. Guenther, C. Hoelbling, S. D. Katz, L. Lellouch, T. Lippert, K. Miura, L. Parato, and K. K. Szabo et al., Nature (London) 593, 51 (2021).

[67] C. Lehner and A. S. Meyer, Phys. Rev. D 101, 074515 (2020).

[68] M. Davier, A. Höcker, B. Malaescu, C. Z. Yuan, and Z. Zhang, Eur. Phys. J. C 74, 2803 (2014).

[69] S. Gonzàlez-Solís and P. Roig, Eur. Phys. J. C 79, 436 (2019).

[70] J. A. Miranda and P. Roig, Phys. Rev. D 102, 114017 (2020).

[71] M. Benayoun, L. Delbuono, and F. Jegerlehner, Eur. Phys. J. C 80, 81 (2020); 80, 244(E) (2020). 
[72] M. Benayoun, L. DelBuono, and F. Jegerlehner, arXiv:2105.13018.

[73] H. n. Li and H. Umeeda, Phys. Rev. D 102, 094003 (2020).

[74] C. Aubin, T. Blum, M. Golterman, and S. Peris, Phys. Rev. D 102, 094511 (2020).

[75] B. Malaescu and M. Schott, Eur. Phys. J. C 81, 46 (2021).

[76] A. Crivellin, M. Hoferichter, C. A. Manzari, and M. Montull, Phys. Rev. Lett. 125, 091801 (2020).

[77] M. Benayoun, P. David, L. DelBuono, and F. Jegerlehner, Eur. Phys. J. C 75, 613 (2015).

[78] G. Colangelo, M. Hoferichter, and P. Stoffer, Phys. Lett. B 814, 136073 (2021).

[79] B. Ananthanarayan, I. Caprini, D. Das, and I. Sentitemsu Imsong, Phys. Rev. D 93, 116007 (2016).

[80] B. Ananthanarayan, I. Caprini, and D. Das, Phys. Rev. D 98, 114015 (2018).

[81] B. Ananthanarayan, I. Caprini, and D. Das, Phys. Rev. D 102, 096003 (2020).

[82] M. Gourdin and E. De Rafael, Nucl. Phys. B10, 667 (1969).

[83] M. Roos, Nucl. Phys. B97, 165 (1975).

[84] C. B. Lang and I. S. Stefanescu, Phys. Lett. 58B, 450 (1975).

[85] R. Omnés, Nuovo Cimento 8, 316 (1958).

[86] A. Pich and J. Portoles, Phys. Rev. D 63, 093005 (2001).

[87] M. Hoferichter, B. Kubis, S. Leupold, F. Niecknig, and S. P. Schneider, Eur. Phys. J. C 74, 3180 (2014).

[88] T. Isken, B. Kubis, S. P. Schneider, and P. Stoffer, Eur. Phys. J. C 77, 489 (2017).

[89] F. Stollenwerk, C. Hanhart, A. Kupsc, U. G. Meissner, and A. Wirzba, Phys. Lett. B 707, 184 (2012).

[90] C. Hanhart, A. Kupśc, U.-G. Meißner, F. Stollenwerk, and A. Wirzba, Eur. Phys. J. C 73, 2668 (2013); 75, 242(E) (2015).

[91] C. Hanhart, Phys. Lett. B 715, 170 (2012).

[92] R. Garcia-Martin, R. Kaminski, J. R. Pelaez, J. Ruiz de Elvira, and F. J. Yndurain, Phys. Rev. D 83, 074004 (2011).

[93] N. N. Achasov and A. A. Kozhevnikov, JETP Lett. 96, 559 (2013).

[94] N. N. Achasov and A. A. Kozhevnikov, Phys. Rev. D 88, 093002 (2013).

[95] I. V. Danilkin, C. Fernández-Ramírez, P. Guo, V. Mathieu, D. Schott, M. Shi, and A. P. Szczepaniak, Phys. Rev. D 91, 094029 (2015).

[96] M. F. Heyn and C. B. Lang, Z. Phys. C 7, 169 (1981).

[97] G. J. Gounaris and J. J. Sakurai, Phys. Rev. Lett. 21, 244 (1968).

[98] J. P. Lees et al. (BABAR Collaboration), Phys. Rev. D 86, 032013 (2012).

[99] J. H. Kühn and A. Santamaria, Z. Phys. C 48, 445 (1990).

[100] C. Hanhart, S. Holz, B. Kubis, A. Kupść, A. Wirzba, and C. W. Xiao, Eur. Phys. J. C 77, 98 (2017).

[101] H. B. O'Connell, B. C. Pearce, A. W. Thomas, and A. G. Williams, Prog. Part. Nucl. Phys. 39, 201 (1997).
[102] J. E. Augustin, J. C. Bizot, J. Buon, J. Haissinski, D. Lalanne, P. Marin, H. Nguyen Ngoc, J. Perez-Y-Jorba, F. Rumpf, E. Silva, and S. Tavernier, Phys. Lett. 28B, 508 (1969).

[103] A. Quenzer, M. Ribes, F. Rumpf, J. L. Bertrand, J. C. Bizot, R. L. Chase, A. Cordier, B. Delcourt, P. Eschstruth, F. Fulda, G. Grosdidier, J. Haissinski, J. Jeanjean, M. Jeanjean, R. J. Madaras, J. L. Masnou, and J. PerezY-Jorba, Phys. Lett. 76B, 512 (1978).

[104] L. M. Barkov, A. G. Chilingarov, S. I. Eidelman, B. I. Khazin, M. Y. Lelchuk, V. S. Okhapkin, E. V. Pakhtusova, S. I. Redin, N. M. Ryskulov, Y. M. Shatunov, A. I. Shekhtman, B. A. Shvarts, V. A. Sidorov, A. N. Skrinsky, V. P. Smakhtin, and E. P. Solodov, Nucl. Phys. B256, 365 (1985).

[105] R. R. Akhmetshin et al. (CMD-2 Collaboration), Phys. Lett. B 527, 161 (2002).

[106] R. R. Akhmetshin et al. (CMD-2 Collaboration), Phys. Lett. B 578, 285 (2004).

[107] V. M. Aul'chenko et al. (CMD-2 Collaboraiton), JETP Lett. 82, 743 (2005).

[108] V. M. Aul'chenko et al., JETP Lett. 84, 413 (2006).

[109] R. R. Akhmetshin et al. (CMD-2 Collaboration), Phys. Lett. B 648, 28 (2007).

[110] B. Aubert et al. (BABAR Collaboration), Phys. Rev. Lett. 103, 231801 (2009).

[111] M. Ablikim et al. (BESIII Collaboration), Phys. Lett. B 753, 629 (2016).

[112] M. Ablikim et al. (BESIII Collaboration), Phys. Lett. B 753, 629 (2016).

[113] A. Aloisio et al. (KLOE Collaboration), Phys. Lett. B 606, 12 (2005).

[114] F. Ambrosino et al. (KLOE Collaboration), Phys. Lett. B 670, 285 (2009).

[115] F. Ambrosino et al. (KLOE Collaboration), Phys. Lett. B 700, 102 (2011).

[116] D. Babusci et al. (KLOE Collaboration), Phys. Lett. B 720, 336 (2013).

[117] A. Anastasi et al. (KLOE-2 Collaboration), J. High Energy Phys. 03 (2018) 173.

[118] M. N. Achasov et al., J. Exp. Theor. Phys. 101, 1053 (2005).

[119] M. N. Achasov et al., J. Exp. Theor. Phys. 103, 380 (2006).

[120] M. N. Achasov et al. (SND Collaboration), J. High Energy Phys. 01 (2021) 113.

[121] S. Gardner and H. B. O'Connell, Phys. Rev. D 57, 2716 (1998); 62, 019903(E) (2000).

[122] G. Barton, Introduction to Dispersion Techniques in Field Theory (Benjamin, New York, 1965).

[123] D. Gómez Dumm and P. Roig, Eur. Phys. J. C 73, 2528 (2013).

[124] R. Aaij et al. (LHCb Collaboration), arXiv:2109.01056.

[125] W. Qin, L. Y. Dai, and J. Portolés, J. High Energy Phys. 03 (2021) 092. 